Zielgruppen ökologischen Konsumverhaltens

\section{Kommunikation gegen den Roll-back}

\begin{abstract}
"Öko ist out!" oder "Ich bin öko-gestresst". Immer häufiger wird man mit derartigen Aussagen konfrontiert, wenn heute das Thema Ökologie zur Sprache kommt. Auch das alternative Milieu, das sich durch die Verbindung von Umweltbewusstsein und -verhalten auszeichnete, ist nahezu verschwunden. Die Frage nach ökologischem Konsumverhalten stellt sich daher völlig nev. Wer ist heutzutage überhaupt noch für ökologischen Konsum ansprechbar? Auf welche Zielgruppen kann sich die Kommunikation nachhaltigen Konsumverhaltens stützen?
\end{abstract}

$\mathrm{D}$

Von Claudia Empacher

festgestellte Rückgang der Begeisterung für das Umweltthema hat viele Gründe. Vor allem die Diskussionen und Skandale um das Duale System Deutschland (DSD) haben bei den Konsumenten und Konsumentinnen ihre Spuren hinterlassen. Die intensive öffentlichkeitsarbeit des DSD hat einerseits dazu geführt, dass das Thema Mülltrennung zum bestkommunizierten Umweltthema überhaupt geworden ist. Andererseits hatte dies jedoch den Effekt, dass sich für viele Konsumenten das Umweltverhalten auf die Mülltrennung beschränkt und andere Konsumbereiche nicht mehr als umweltrelevant wahrgenommen werden. Die Aufdeckung der Müllskandale bewirkte in der Folge nicht nur eine Image-Schädigung des dualen Systems, sondern griff auf den gesamten Umweltbereich über. Immer häufiger wird die Meinung vertreten, das Thema Umwelt sei ohnehin nur Schwindel, mit dem der Verbraucher ,abgezockt" werden soll. Dennoch können die schwarzen Schafe und Skandale im Öko-Bereich nicht allein für das wachsende Desinteresse verantwortlich gemacht werden. Auch auf Seiten der Umweltkommunikation sind zahlreiche Fehler gemacht worden. Vor allem zu Beginn der Umweltdiskussion wurde sehr moralisierend argumentiert und zu stark auf Verzicht als Lösungsstrategie gesetzt. Obwohl dieser Vorwurf heute so nicht mehr gilt, steht das Umweltthema nach wie vor unter Ideologie-Verdacht und leidet unter einem asketischen und lustfeindlichen Image. Gerade Askese und Verzicht sind jedoch zu Beginn des neues Jahrtausends keine attraktiven Lebensziele mehr. Weder
Abfall- und Umweltberatung noch die Anbieter ökologischer Produkte haben es bisher geschafft, das Thema modern und attraktiv zu vermitteln. Daneben gibt es aber auch andere gesellschaftliche Entwicklungen, die den Rückgang des ökologischen Problembewusstseins mitbewirkt haben, wie etwa die hohe Arbeitslosigkeit oder die Erosion sozialer Sicherungssysteme. Welche Chancen bestehen angesichts dieser Entwicklungen überhaupt noch, Umweltverhalten wieder zum Thema zu machen?

\section{An den Zielgruppen für ökologi- sches Konsumverhalten ansetzen}

In der Fachdiskussion hat sich inzwischen die Erkenntnis durchgesetzt, dass die Bereitschaft zu ökologischem Konsum bei verschiedenen Konsumentengruppen und Lebensstilen ganz unterschiedlich ausgeprägt ist (2). Diese Potenziale gilt es zu identifizieren und Kommunikationssowie Maßnahmestrategien für unterschiedliche Zielgruppen zu entwickeln.

Bereits existierende Lebensstiltypologien der Sozial-, Markt- und Meinungsforschung, wie beispielsweise die sozialen Milieus von SINUS (3), differenzieren allerdings nicht näher hinsichtlich ökologischer Verhaltenspotenziale und können daher für eine Ausrichtung von Umweltkommunikation auf Zielgruppen und ein umweltorientiertes Marketing nur geringe Anhaltspunkte liefern. Eine Konkretisierung dieser Typologien auf die Bereitschaft hin, nachhaltigen Konsum zu praktizieren, wurde bisher erst in einzelnen Verbrauchsfeldern, vor allem bezogen auf den Energieverbrauch (4), oder in einzelnen Bedürfnisfeldern, z.B. Mobilität (5), vorgenommen.
Vom Institut für sozial-ökologische Forschung (ISOE) wird zur Zeit ein Projekt mit dem Titel „Haushaltsexploration zu den Bedingungen, Möglichkeiten und Grenzen nachhaltigen Konsumverhaltens" durchgeführt, das versucht, diese Lücke zu schließen. Es stellt ökologische Handlungspotenziale bezogen auf den gesamten Konsumbereich privater Haushalte in den Mittelpunkt (6). Das Ziel des Projektes besteht darin, zielgruppenspezifische Strategien für nachhaltiges Konsumverhalten zu entwickeln.

Ohne bereits auf genauere Ergebnisse eingehen zu können, lassen sich hier einige Tendenzen identifizieren, die eine Herausforderung für die Umweltkommunikation und umweltorientiertes Marketing darstellen, gleichzeitig aber auch Anknüpfungspunkte und Potenziale für die Ansprache neuer Zielgruppen bieten.

\section{Zielgruppen: Überforderte ...}

Die wachsende Schere zwischen Arm und Reich - unter anderem verursacht durch steigende Arbeitslosigkeit, sinkende Reallöhne und mangelnde soziale Absicherung - lässt eine immer größer werdende Gruppe von finanziell Überforderten entstehen. Diese Personen leben nicht unbedingt von der Sozialhilfe, aber ihr finanzielles Polster ist so gering, dass sie ständig gegen den sozialen Abstieg kämpfen. Neben der finanziell angespannten Lage erfordert dieser Kampf auch einen hohen Einsatz an Zeit und Energie. Diese Gruppe nimmt das ganze Umweltthema, insbesondere auch ökologische Produkte, als Luxus wahr, für die sie weder Geld noch Zeit hat.

Gleichzeitig ist bei dieser Gruppe der Informationsstand über einfache Verhaltensweisen des Ressourcensparens, zum Beispiel bezüglich Energie und Wasser, sehr gering. Aufgabe der Umweltkommunikation wäre es, diesen Überforderten einfache Verhaltensweisen und Angebote zu vermitteln, wie durch Umweltverhalten auch Geld gespart werden kann. Hierzu ist es zunächst jedoch notwendig, bestehende Barrieren abzubauen. Dies gilt insbesondere für die in der Gruppe vorherrschende Vorstellung, das Umweltthema sei etwas Abgehobenes für Intellektuelle. So müsste die Umweltberatung auch ihre Kommunikationsmedien und die Art der Kommunikation überdenken. Umweltkommunikation arbeitet immer noch zu sehr kognitiv-rational, teilweise sogar in wissenschaftlicher Terminologie. Vieles wird schriftlich in naturwissenschaftlicher Fachsprache abstrakt ausge- 
drückt. Die Anschaulichkeit von Maßnahmen zum Umweltverhalten und das Anknüpfen an Alltagserfahrungen ist jedoch nicht nur für die Zielgruppe der Überforderten attraktiv, sondern bietet auch für andere Gruppen eine geeignetere Zugangsform.

\section{>... Winner-Typen ...}

Den Gegenpol zu den Überforderten repräsentiert eine andere Gruppe, die gleichsam den WinnerTyp in unserer Gesellschaft darstellt. Hierbei handelt es sich um noch relativ junge, beruflich erfolgreiche Leute mit hohem Einkommen. Der Typ ist Trendsetter nicht nur in Bezug auf die Produkte, die von ihm konsumiert werden, sondern auch hinsichtlich der Werte, die er verkörpert. Modernität, Individualität und Professionalität zeichnen diesen Typus aus, verbunden mit einer positiven Lebenseinstellung, in der Spaß, Action und bewusst inszenierte Erlebnisse eine große Rolle spielen. Beim Konsumverhalten sind ihm Design und Ästhetik der Produkte wichtig. Im Gegensatz zu dem Ende der 80er Jahre aufgekommenen Bild des Yuppie, der sich von allen sozialen Verpflichtungen gelöst zu haben schien und der beruflich als skrupellos galt, fühlt sich der moderne Winner-Typ durchaus gewissen sozialen Werten verpflichtet. Auch die Bedeutung ökologischer Probleme erkennt er an, allerdings grenzt er sich als liberal denkender Mensch deutlich zur "Öko-Ideologie“ ab. Ökologische Produktangebote findet er grundsätzlich begrüßenswert, jedoch gefallen ihm weder Design noch Ästhetik. Er empfindet ökologische Verhaltensangebote als zu langsam und zu umständlich und den Service als nicht schnell und professionell genug. Mit anderen Worten: Öko ist für ihn $\mathrm{zu}$ wenig modern.

Die gesellschaftliche Vorbildfunktion dieser Gruppe zu nutzen, wäre für eine Beförderung ökologischen Konsumverhaltens von hoher Bedeutung. Um sie erreichen zu können, muss sich jedoch das Umweltthema seines AlternativImages entledigen. Öko soll Spaß machen und modern sein. Hierzu müssen die ökologischen Produkt- und Verhaltensangebote den Touch des Selbstgemachten und Unprofessionellen verlieren, statt dessen sollten Design, Mode und Service im Mittelpunkt stehen. Entsprechende Angebote gilt es jedoch erst noch zu entwickeln, unter Umständen sind hier auch ökologische Kompromisse unumgänglich. Bezüglich des Marketing ist zudem Vorsicht geboten, da das Umweltargument nicht in den Vordergrund gestellt werden darf. Mode-Anbieter wie Britta
Steilmann, die den Aufbau einer Öko-Kollektion wagten, scheiterten an diesen falschen Marketing-Strategien.

\section{Traditionalisten ...}

Nach wie vor existieren jedoch auch große Gruppen in unserer Gesellschaft, die traditionellen Werten eine große Bedeutung beimessen oder sich wieder verstärkt auf diese besinnen. Hierzu gehören zum Einen wertkonservative Gruppen, die bildungsbürgerlichen Milieus entstammen. Ihnen sind Werte wie Nähe, Freunde und Familie, Erhaltung und Bewahren von Traditionen sowie Qualität von Waren und Dienstleistungen wichtig.

Zum Anderen finden sich im traditionellen Segment Vertreter kleinbürgerlicher Milieus, die an Werten wie Disziplin, Pflichterfïllung und Verlässlichkeit festhalten, bei denen aber auch Nähe, soziale Einbettung und Nachbarschaftshilfe sowie die Schaffung bleibender Werte und Besitz von Bedeutung sind. Beide Gruppen sind aufgrund ihrer Wertestruktur gegenüber dem Konzept der Nachhaltigkeit und dem Kreislaufgedanken aufgeschlossen, sie lehnen jedoch Öko als (vermeintlich linke) Ideologie stark ab. Offen für nachhaltiges Konsumverhalten sind sie, wenn es um die Erhaltung und den Schutz der Natur oder um

\title{
punkt-um.net
}

\section{Ihre Internet-Plattform zum Infodienst punkt.um für Informationen zu Nachhaltigkeit und Ökologie}

\author{
Aktuelle Termine - News aus dem Internet - Web-Site-Datenbank \\ OekoDok: Abstracts-Datenbank zu 60.000 Zeitschriftenartikeln seit 1987
}

Sie finden uns unter: http://www.punkt-um.net 
sozialen Zusammenhalt geht. Für Produkte aus fairem Handel sind diese Gruppen ebenso ansprechbar wie für qualitativ hochwertige und langlebige Produkte.

\section{... und die Ex-Alternativen}

Zu guter Letzt existiert immer noch eine Gruppe, bei der wichtige ökologische Konsumverhaltensweisen zur Routine und damit selbstverständlich geworden sind. Beispiele sind die Mülltrennung, der Kauf von Recycling-Produkten, eine häufige Nutzung des öffentlichen Nahverkehrs und umweltschonendes Waschverhalten. $\mathrm{Zu}$ dieser Gruppe zählen vor allem die ehemals Alternativen und verwandte soziale Milieus, die heute weniger alternativ, sondern eher etabliert sind und sich neuen Verpflichtungen gegenüber sehen. Häufig haben die Vertreter dieser Gruppe inzwischen Familien gegründet. Gleichzeitig versuchen sie den emanzipatorischen Anspruch in ihrem Familienleben umzusetzen, so dass beide Partner berufstätig sind. Zusammen mit den Kindern erfordert diese Doppelbelastung einen erheblichen Organisationsaufwand innerhalb der Familie und führt zu großer Zeitknappheit. Dementsprechend bestehen hohe Convenience-Ansprüche an Produkte sowie die Gefahr, dass ökologische Verhaltensweisen, die als zu zeitaufwendig empfunden werden, zugunsten der Haushalts-Rationalisierung aufgegeben werden. Der Anspruch, sich ökologisch zu verhalten, wird zwar aufrecht erhalten, aber mit jeder zusätzlichen Belastung heruntergeschraubt.

\section{Sozial-ökologische Unternebmens- bewertung}

und

\section{Nachbaltige Landwirtschaft}

sind die Themen der Informationsdienste

$$
\begin{gathered}
\text { Ökologisches } \\
\text { Wirtschaften 2/2000 } \\
\text { und 3-4/2000 }
\end{gathered}
$$

Wenn Sie potenzielle Beiträge haben, wenden Sie sich bitte an die Redaktion.
Für diese Gruppe gilt es somit, zeitsparende ökologische Verhaltensangebote zu entwickeln, um ihr Umweltengagement aufrecht zu erhalten.

Obwohl die bisher aufgezeigten Tendenzen durchaus Potenziale für ökologischen Konsum beinhalten, zeigen sich auf der anderen Seite auch die Grenzen für das Umweltthema.

\section{Grenzen von}

\section{Zielgruppenstrategien}

Seit dem Aufkommen der Umweltbewegung wurde sehr starkes Gewicht auf die Aufklärung des mündigen Bürgers über die Umweltzusammenhänge und Problemursachen gelegt. Demgegenüber kann jedoch inzwischen bei einer größeren Gruppe von Verbrauchern die Tendenz festgestellt werden, überhaupt nicht mehr alles wissen zu wollen und nicht mehr alle Zusammenhänge verstehen zu müssen (7). Aufgrund von Überforderung und Informationsüberflutung beginnen Konsumenten, sich bewusst von dem Anspruch abzugrenzen, alles selbst wissen und entscheiden zu müssen und damit auch die volle Verantwortung für ihr Handeln zu übernehmen. Statt dessen wird vermehrt der Anspruch geäußert, bestimmte Eigenschaften von Produkten, wie die Umweltverträglichkeit, müssten vom Hersteller oder durch politische Rahmenbedingungen garantiert sein, so dass sich der Verbraucher keine weiteren Gedanken darüber machen muss. Ähnlich wie im Öko-Bereich dem Naturkosthandel hinsichtlich der Erfüllung ökologischer Kriterien fast blind vertraut wird, vertrauen andere Zielgruppen bestimmten Markenherstellern (8). Angesichts einer immer komplexeren Umwelt, undurchschaubarer Problemzusammenhänge und einer Flut unterschiedlicher Informationen hat dieser Anspruch des Nicht-Wissen-Wollens und das Zurückverweisen der Verantwortung auf entsprechende Experten durchaus seine Berechtigung. Es gilt also, Lösungen zu entwickeln, die die Verbraucherinnen und Verbraucher entlasten. Ein Beispiel sind Produkt- und Dienstleistungsangebote, deren Umweltnutzen sich automatisch realisiert und nicht erst umständlich vermittelt werden muss.

Zusammenfassend lässt sich feststellen, dass es nach wie vor erhebliche Potenziale für ökologisches Konsumverhalten bei einigen Zielgruppen gibt. Um diese für ökologischen Konsum fruchtbar machen zu können, müssen sich allerdings sowohl die Art der Umweltkommunikation als auch die Angebote nachhaltiger Konsumverhaltensweisen ändern. Umweltkommunikation und ökologisch orientierte Anbieter müssen sich den aktuellen gesellschaftlichen Trends stellen und offensiv neue Angebote entwickeln, anstatt am überholten ÖkoImage festzuhalten und über den Rückgang des Umweltbewusstseins zu lamentieren. Auch wenn hierbei vielleicht mit dem einen oder anderen Tabu gebrochen werden muss, ist eine moderne Version des Umweltthemas besser als eine, die keine Anhänger mehr findet.

\section{Anmerkungen}

(1) Vgl. Bundesministerium für Umwelt, Naturschutz und Reaktorsicherheit ( $\mathrm{Hg}$. ): Umweltbewusstsein in Deutschland 1998. Berlin 1998.

(2) Vgl. u.a. Gillwald, K.: Ökologisierung von Lebensstilen - Argumente, Beispiele, Einflussgrößen. wzb-paper FS II 95-408, Berlin 1995 sowie Schultz, I./ Weller, I.: Nachhaltige Konsummuster und postmaterielle Lebensstile. Vorstudie im Auftrag des Umweltbundesamtes. Berlin: UBA-Texte $30 / 1997$.

(3) Vgl. SINUS: Lebenswelfforschung und Soziale Milieus in West- und Ostdeutschland. Heidelberg 1993 sowie SINUS: Die SINUS-Milieus. Kurzinformation. Heidelberg 1999.

(4) Vgl. Reusswig, F.: Lebensstile und Ökologie. Sozialökologisches Arbeitspapier 43. Frankfurt/M. 1994; Schoenheit, I./ Niedergesäß, U.: Lebensstile und Energieberatung, Hannover 1995 und Prose, F./ Wortmann, K.: Energiesparen: Verbraucheranalyse und Marktsegmentierung der Kieler Haushalte. Unveröffentlichtes Manuskript. Kiel 1991.

(5) Vgl. ISOE: Mobilitätsstile. Ein sozial-ökologischer Untersuchungsansatz. Forschungsbericht "Stadtverträgliche Mobilität", Band 7. Frankfurt/M. 1997.

(6) Das Projekt ist das zweite Teilprojekt eines vom Umweltbundesamt initiierten Demonstrationsvorhabens zur Fundierung und Evaluierung nachhaltiger Konsummuster und Verhaltensstile (siehe Projektdarstellung S. 26). Ergebnisse des Projektes können voraussichtlich Mitte 2000 beim Umweltbundesamt angefordert werden.

(7) Vgl. Götz, K.: Ansprüche an ökologische Innovationen im Textilbereich. ISOE DiskussionsPapier 11. Frankfurt/M. 1999.

(8) Vgl. u.a. Empacher, C./ Götz, K.: Ansprüche an ökologische Innovationen im Lebensmittelbereich. ISOE DiskussionsPapier 10. Frankfurt/M. 1999.

\section{Die Autorin}

Claudia Empacher ist wissenschaftliche Mitarbeiterin am Institut für sozial-ökologische Forschung im Forschungsbereich Stoffströme und Alltagsökologie. Kontakt: ISOE, Hamburger Allee 45, 60486 Frankfurt/M., Tel. 069/ 7076919-20, E-mail: empacher@isoe.de 
(c) 20I0 Authors; licensee IÖW and oekom verlag. This is an article distributed under the terms of the Creative Commons Attribution Non-Commercial No Derivates License (http://creativecommons.org/licenses/by-nc-nd/3.o/), which permits unrestricted use, distribution, and reproduction in any medium, provided the original work is properly cited. 